\title{
M2DFR: Multi-layer Multipath Data Forwarding Routing Protocol for Underwater Wireless Sensor Network
}

\author{
Mukhtiar Ahmed*, Nadeem Naeem, Sajida Parveen, Nazar Hussain and Rajab Malookani \\ Quaid-e-Awam University, Engineering, Science and Technology, Sindh, Pakistan; mukhtiar.a@gmail.com, \\ nadeemnaeem@yahoo.com, nadeemnaeem@yahoo.com, \\ nazarphulpoto@quest.edu.pk,rajab_ali31@yahoo.com
}

\begin{abstract}
Objectives: To design and develop the Multi-layer Multipath Data Forwarding Routing (M2DFR) protocol for Underwater Wireless Sensor Network which controls the water depth, node mobility and can enhance the overall performance. Method/Analysis: M2DFR is based on three phases, one is design of architecture under which the deployment of the nodes are shown, second is the initialization phase which develops the multipath route selection mechanism between seabed source nodes to ordinary nodes which are deployed in the lower depth to upper layer, Acoustic Powerful Nodes (APNs) deployed in upper depth in fixed way in layers to surface sink nodes which are deployed on water surface, the route selection for multipath is based on RREQ/RREP through Weight Calculation Mechanism (WCM). Third phase focuses the data forwarding phase under which the shortest WCM is selected and from this route packets are forwarded from source nodes to sink nodes. Findings: Most of the existing protocols are unable to overcome the problem of water depth controlling, uncontrolled node mobility. Applications/Improvements: M2DFR is designed to extract the information from the bottom of the sea with its applications like: gold, oil/gas and minerals. The improved performance of M2DFR is measured with CBE2R and EMGGR.
\end{abstract}

Keywords: APNs, Higher-Depth, Lower-Depth, Node-Mobility, WCM

\section{Introduction}

To design the routing protocol in underwater environment is one of the challenging tasks, because in underwater environment there is majority number of issues like: Water pressure, acoustic channel limitation, sensor node battery power problem, long distance from water surface to seabed, water current, 3D deployment and uncontrollable node mobility ${ }^{\underline{1}-4}$. Designing of routing protocol in underwater environment is the major focus of the network based researchers due to its valuable application based information like: finding of coal mines, gold, oil/gas, minerals, diamond etc ${ }^{\underline{5}-7}$. In underwater environment the four types of the nodes are used, one is sink node which almost is deployed on water surface, the courier nodes or underwater vehicles which are deployed in underwater environment, the ordinary nodes which are also deployed in underwater and the source nodes which are almost deployed at the seabed level ${ }^{-12}$. The acoustic channel is used for packets forwarding because the RF and fiber optical based channel can't work properly in underwater environment due to its limitations in underwater environment ${ }^{13}-\underline{16}$. In underwater environment the water depth controlling, deployment of nodes and controlling of node mobility still needs improvement, though majority of the routing protocols are designed for 
controlling of these issues. This research article presents the Multi-layer Multipath Data Forwarding Routing (M2DFR) protocol which controls the water depth, node mobility and deployment issues. Detailed discussion of the M2DFR is presented in Section 3.

\section{Related Work}

In this section we present the operation of the related routing protocol which resembled with M2DFR. The problems faced by existing routing protocols are also mentioned in this section.

Hop-by-Hop Depth Addressing Based Routing (H2-DAB) protocol as mentioned in ${ }^{17}$ controls the water depth with dynamic addressing mechanism to the nodes from seabed to water surface. H2-DAB deploy the nodes from sea surface to seabed with some usage of courier nodes. 3D deployment mechanism has been adapted by $\mathrm{H} 2-\mathrm{DAB}$. The route development mechanism is based on depth addressing mechanism. It is observed from the operation of $\mathrm{H} 2$-DAB that when network becomes sparse due to water pressure and node mobility the overall performance becomes reduced.

Multi-layer Routing Protocol (MRP) as given in $\frac{18}{}$ controls the water depth through formation of layers, MRP deployed the super nodes in upper and lower depth of water, the authors of the MRP deployed the ordinary nodes at the lower depth of the water, the layering addressing ID mechanism is used for to route the packets from source nodes which are deployed at seabed to sink nodes which are deployed on water surface. MRP loses its performance due to uncontrollable node mobility because due to small interest area the node mobility resets the network in couple of minutes.

Energy-efficient Multipath Grid-based Geographic Routing (EMGGR) protocol controls the water depth through formation of the multiple $3 \mathrm{D}$ grids ${ }^{19}$. The protocol operation is based on the three phases, these phases are: gateway election mechanism, updating of gateways election mechanism, and data forwarding. For data forwarding the nodes are deployed in different cells of 3D grid under which the gateway election for particular cell is to be elected by xyz addressing mechanism and through creation of the multipath the data packets are forwarded from source to sink node. EMGGR focuses the formation of the $3 \mathrm{D}$ grids in underwater environment is just hypothesis, in real scenario such kind of methodology is not possible due to node mobility and underwater pressure.

Clustered Based Energy Efficient Routing (CBE2R) protocol as discussed in 2 . CBE2R control the water depth through use of courier nodes and formation of layers from water surface to seabed. The authors of the CBE2R have deployed the ordinary nodes at the bottom layer of the water and deployed the ordinary nodes at the bottom layer towards seabed, the courier nodes has been deployed in fixed way at each layer, whereas the multiple sink nodes are deployed on the water surface. The packets forwarding mechanism of the CBE2R is based on the cluster formation with lower weight values. It is observed that when network becomes sparse the performance of $\mathrm{CBE} 2 \mathrm{R}$ is not reasonable.

Reliable Multipath Energy Efficient Routing (RMEER) protocol also controls the water depth through multiple layer formation mechanism and use of static courier nodes ${ }^{3}$. The multipath packets forwarding mechanism has been adapted at seabed level from source to static courier nodes to sink nodes. Source nodes are deployed at the seabed level whereas courier nodes are deployed on multiple layers in fixed way from sea surface to seabed. Courier nodes are the powerful nodes and having high battery power, courier nodes collects the information from source nodes through ordinary nodes and forwards that information towards sink nodes which are deployed on sea surface. In RMEER the deployment of the fixed courier nodes throughout the water from sea surface to seabed is the complicated mechanism and is expensive one. RMEER only works on vertical modem; if network becomes sparse then there is need of the horizontal modem; so the performance of RMEER in sparse network is not so reasonable.

\section{Multi-layer Multipath Data Forwarding Routing (M2DFR)}

This section focuses the proposed routing protocol M2DFR with proposed architecture, initialization phase, and data forwarding mechanism.

\subsection{Network Architecture}

Figure 1 focuses the network architecture of the M2DFR. In M2DFR, the water depth of the sea water which is $15 \mathrm{Km}$ from sea surface to seabed is divided into upper depth $(12 \mathrm{Kms})$ and the lower depth $(3 \mathrm{Kms})$. In upper 
depth the 3 layers are formed with equal size of the 4 Kms and the fixed Acoustic Powerful Nodes (APNs) are deployed on each layer in fixed way. The sink nodes which are the destination nodes are deployed on the water surface and are connected between each other through Radio Frequency signaling (RF). Sink nodes collects the application based data from courier nodes and forward that data to the data storage center. The source nodes are deployed at the seabed which collects the information from the seabed and forward it to the APNs through ordinary nodes. Ordinary nodes make the multipath between source nodes to upper depth layer of APNs as shown in Figure 1. In underwater environment the acoustic channel is used to forward the data packets. The acoustic modem covers the vertical and horizontal data forwarding mechanism.

\subsection{Initialization Phase}

In this phase the multipath between source nodes and APNs has been developed through ordinary nodes. The source nodes are placed at the seabed where as ordinary nodes are deployed in lower depth of water. If source nodes have the data packets then source node will forward the RREQ packet to all the ordinary nodes and when all the nodes will receive the RREQ, the every node will forward the RREP to the source node after making the multipath between bottom layers of APNs from the upper depth. Every route which is developed between source nodes to APNs is based on Weight Calculation Mechanism (WCM). WCM is based on distance (d) and minimum number of hops (hs). The WCM is shown in Equation 1.

$$
W C M=d+s \quad \text { Equation (1) }
$$

The multipath development mechanism and WCM assigning mechanism is shown in Figure 2.

\subsection{Data Forwarding Mechanism}

The data forwarding mechanism is based on the route selection with shortest WCM as shown in Figure 2. The WCM is assigned to every link after forwarding the RREQ and RREP from source to APNs. The source node will look the shortest WCM during forwarding of the RREQ and receiving the RREP. The shortest WCM for packets forwarding is shown in Figure 3.

From Figure 2, after the assigning of the WCM which is based on d in meters and hs (hops). The calculation mechanism is based on minimum number of hops and the shortest distance. Figure 3 focuses the route selection w.r.t minimum WCM which is:

$$
\text { Source } \rightarrow 5 \rightarrow 4 \rightarrow A P N
$$

After the shortest route selection through WCM, the source nodes will forward the data packets through shortest route, when APNs receive the data packets, the APNs with it highly Power Generation Mechanism (HPM) will forward the data packets to upward APNs and in this way the data packets will be received by the surface sink nodes. From sink nodes the data further forwarded to the data storage center. M2DFR specially is designed for collection of gold, oil/gas and valuable minerals base data.

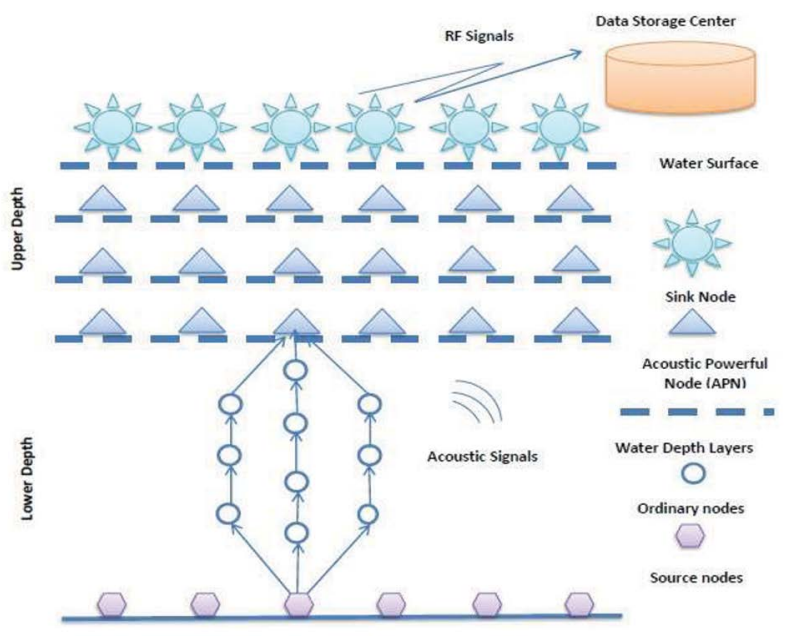

Figure 1. Network architecture of M2DFR.

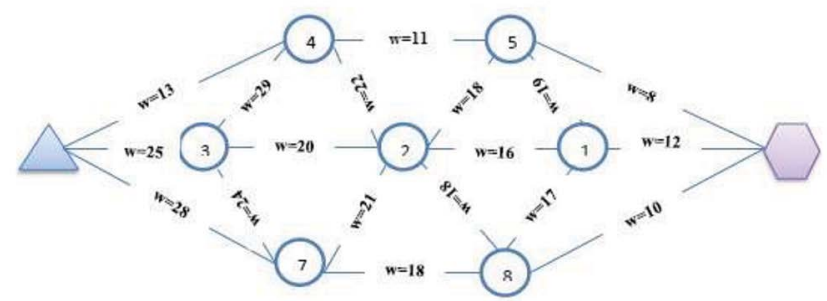

Figure 2. Route assigning mechanism through WCM.

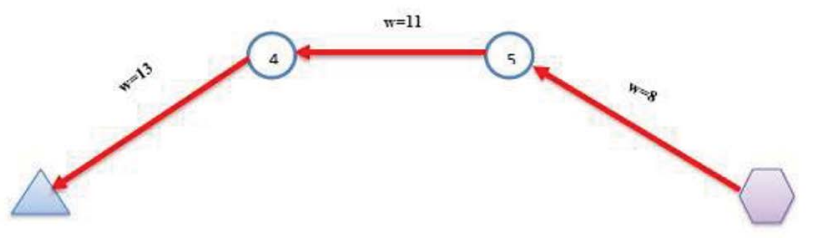

Figure 3. Shortest route from source to APNs. 


\section{Performance Analysis}

M2DFR is benchmarked with CBE2R and EMGGR. The performance analysis is measured in terms of network throughput, packets delivery ratio, network lifetime and end-to-end delay. For performance analysis the NS2.30 with AquaSim package simulator is used. The NS2.30 with AquaSim is set on parameters which are shown in Table 1.

\subsection{Network Throughput}

Network throughput can be measured in kilobits per seconds and is the cumulative response of entire network data received by sink nodes. Network throughput of M2DFR is shown in Figure 4.

In Figure 4, the M2DFR is compared with CBE2R and EMGGR.

In Figure 4, that network throughput of M2DFR is higher than CBE2R and EMGGR because M2DFR controls the water in efficient way due to division of water depth into upper and lower. The use of APNs also enhances

Table 1. Simulation setup

\begin{tabular}{lll}
\hline S/No & Parameters & Value \\
\hline 1. & Network Size & $2000 \mathrm{~m} \times 2000 \mathrm{~m} \times 2000 \mathrm{~m}$ \\
2. & Total nodes & 300 \\
3. & $\begin{array}{l}\text { Total distance from } \\
\text { water surface to seabed }\end{array}$ & $15 \mathrm{Km}$ \\
4. & Upper depth layer & $4 \mathrm{Kms}$ \\
& distance & \\
5. & Lower depth distance & $3 \mathrm{Kms}$ \\
6. & Modem used (MAC) & $802.11-\mathrm{DYNAV}$ \\
7. & Number of layers & 3 \\
8. & Transmission range & $100 \mathrm{~m} \mathrm{to} 150 \mathrm{~m}$ \\
9. & Simulation time & $1000 \mathrm{sec}$ \\
\hline
\end{tabular}

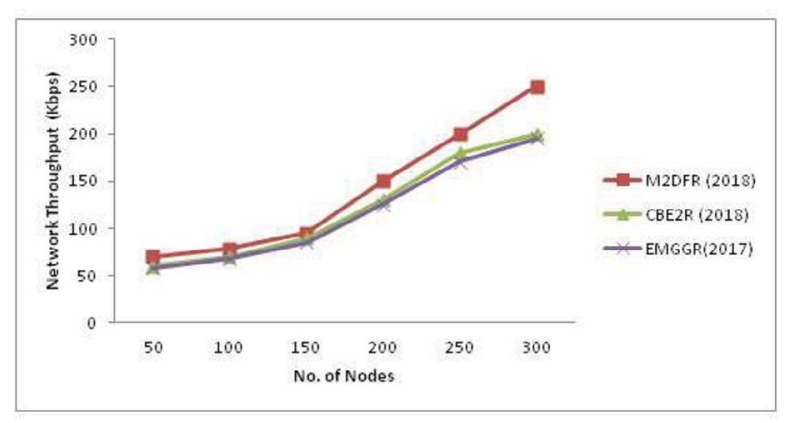

Figure 4. Network throughput (Kbps) of M2DFR. the network throughput due to its HPM. On the other hand the network throughput of CBE2R and EMGGR is lower than M2DFR because CBE2R is only based on vertical modem and forward the data only vertical manner, it observed from the performance of the CBE2R that when due to water pressure network becomes sparse the overall network throughput becomes degraded. EMGGR response in network through put is slow due to gateway election mechanism and formation of the $3 \mathrm{D}$ grids because both are time consuming.

\subsection{Packets Delivery Ratio}

Packets delivery ratio means the ratio between data send from source nodes and data received by the sink nodes. The PDR is measured in \%, the PDR for M2DFR is shown in Figure 5.

In Figure 5 the PDR of M2DFR is higher than CBE2R and EMGGR because the M2DFR uses the stable multipath route selection mechanism with less number of overheads. The WCM enhances the PDR of M2DFR. On other hand the packets PDR for CBE2R and EMGGR is lower than M2DFR because CBE2R only rely on assumed weight values from source to sink node which reduces the PDR. The PDR of EMGGR is lower than proposed protocol because it uses the 3D grid formation mechanism which is not the stable mechanism especially for underwater environment.

\subsection{Network Lifetime}

Network lifetime is defined when any ordinary node or APN die earlier due to energy depletion. The network lifetime can be measured in seconds. The network lifetime of M2DFR is shown in Figure 6.

The network lifetime of M2DFR is higher than CBE2R and EMGGR because in M2DFR the use of APNs

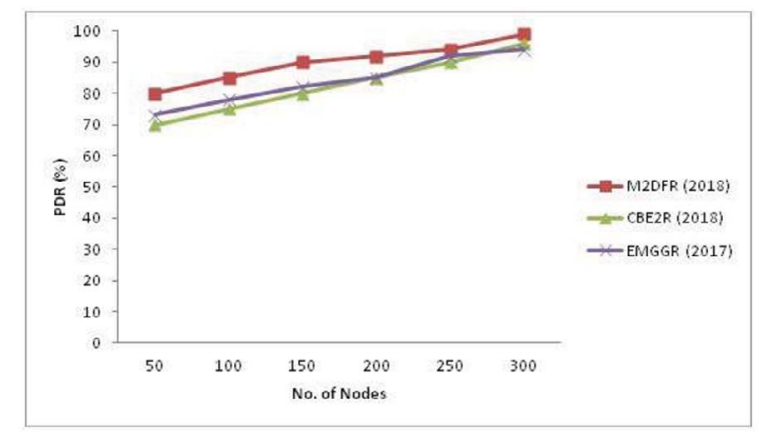

Figure 5. PDR(\%) for M2DFR over CBE2R and EMGGR. 
and WCM enhances the network lifetime of proposed protocol. The network lifetime of CBE2R is lower than M2DFR because CBE2R uses the poor node selection mechanism through weight value mechanism under which node moves every time from its data forwarding route due to node mobility and will drop the packets and will die early. On other hand the network lifetime of EMGGR is lower than proposed protocol because water pressure affects the $3 \mathrm{D}$ grid formation mechanism.

\subsection{Average End-to-End Delay}

Average end-to-end delay is defined the delay at the sink nodes for arriving of packets from all the source nodes. The average end-to-end delay is measured in seconds. The average end-to-end delay of M2DFR is shown in Figure 7.

In Figure 7, the average end-to-end delay of M2DFR is lower than CBE2R and EMGGR because the use of fast APNs with HPM reduces the average end-to-end delay. On other hand the end-to-end delay of CBE2R is higher than proposed protocol because the weight value and cluster formation mechanism takes the highly time. The EMGGR end-to-end delay is higher than proposed

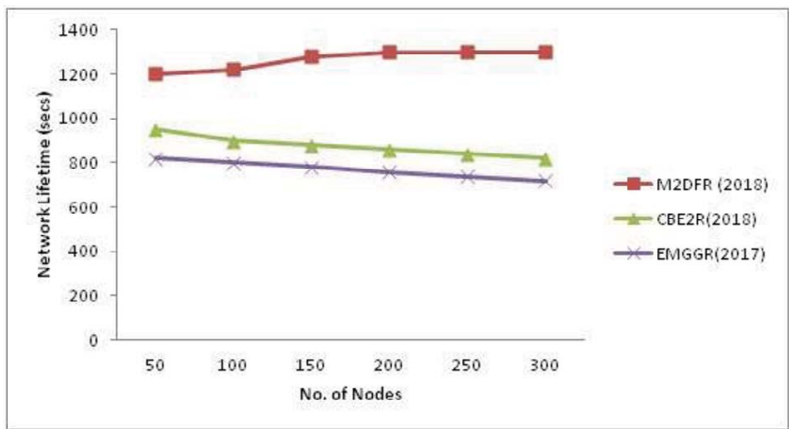

Figure 6. Network lifetime of M2DFR over CBE2R and EMGGR.

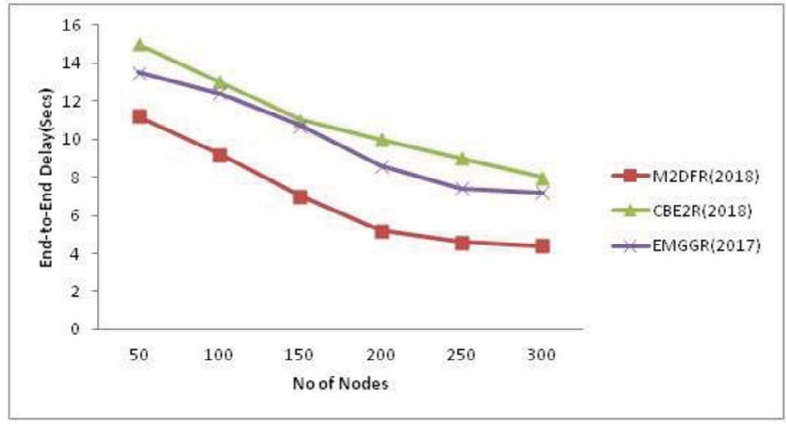

Figure 7. End-to-end delay (Secs) of M2DFR over CBE2R and EMGGR. protocol because the $3 \mathrm{D}$ grid formation mechanism and gateway election mechanism within cells takes the larger time.

\section{Conclusion}

This research article presents the novel designing of M2DFR proposed protocol; the operation of the proposed protocol is based on network architecture, initialization and data forwarding phases. In network architecture the deployment of the sink nodes, APNs, ordinary nodes and source nodes is presented with upper depth and lower depth of water. The initialization phases focus the multipath route development mechanism through RREQ/ RREP and WCM. Through WCM the values are assigned to every link between source nodes to APNs through ordinary nodes. In data forwarding phase the data is forwarded by selected shortest route through WCM from source to APNs to sink nodes. The performance analysis of M2DFR is based on network throughput, PDR, network lifetime, and average end-to-end delay. The performance analysis of M2DFR is better than CBE2R and EMGGR.

\section{References}

1. Ali B, Sher A, Javaid N, Aurangzeb K, Haider SI. Retransmission avoidance for reliable data delivery in Underwater WSNs. Sensors. 2018; 18(1):1-149. https://doi. org/10.3390/s18010149

2. Ahmed M, Salleh M, Channa MI. CBE2R: Clustered-based Energy Efficient Routing protocol for Underwater Wireless Sensor Network. International Journal of Electronics. 2018; 105(11):1916-30. https://doi.org/10.1080/00207217.2018.1 494323

3. Ahmed M, Salleh M, Channa MI, Rohani MF. RMEER: Reliable Multi-path Energy Efficient Routing Protocol for Underwater Wireless Sensor Network. IJECE. 2018; $8(6): 1-9$.

4. Goyal N, Dave M, Verma AK. A novel fault detection and recovery technique for cluster-based Underwater Wireless Sensor Networks. International Journal of Communication Systems. 2018; 31(4):1-14. https://doi.org/10.1002/ dac. 3485

5. Gomathi R, Manickam JML. Energy efficient shortest path routing protocol for underwater acoustic Wireless Sensor Network. Wireless Personal Communications: An International Journal. 2018; 98(1):843-56. https://doi. org/10.1007/s11277-017-4897-5 
6. Gul S, Jokhio SH, Jokhio IA. Light-weight depth-based routing for Underwater Wireless Sensor Network. International Conference on Advancements in Computational Sciences (ICACS); 2018. p. 1-7. PMCid: PMC5901503. https://doi. org/10.1109/ICACS.2018.8333483

7. Wen J, Li D, Liu L, Yuan J. An estimated Hungarian method for data forwarding problem in Underwater Wireless Sensor Networks. International Journal of Distributed Sensor Networks. 2018; 14(5):1-11. https://doi. org/10.1177/1550147718772538

8. Ahsan M, Ahmed S, Hadi F, Wahab F, Ahmed I. A Recent study on routing protocols in UWSNs. International Journal of Advanced Computer Science and Applications. 2017; 8(4):431-5. https://doi.org/10.14569/ IJACSA.2017.080457

9. Zahoor A, Javaid N, Akbar M, Khan ZA. A new routing protocol for maximum coverage in square field for Underwater WSNs. 31st IEEE International Conference on Advanced Information Networking and Applications; 2017. p. 272-7. https://doi.org/10.1109/AINA.2017.154

10. Ahmed M, Salleh M, Channa MI. Routing protocols based on node mobility for Underwater Wireless Sensor Network (UWSN): A survey. Journal of Network and Computer Applications. 2017; 78:242-52. https://doi.org/10.1016/j. jnca.2016.10.022

11. Coutinho RW, Boukerche A, Vieira LF, Loureiro AA. Geographic and opportunistic routing for Underwater Sensor Networks. IEEE Transactions on Computers. 2016; 65(2):548-61. https://doi.org/10.1109/TC.2015.2423677

12. Ahmed M, Salleh M, Channa MI. Critical analysis of data forwarding routing protocols based on single path for UWSN. International Journal of Electrical and Computer Engineering. 2016; 6(4):1695-701. https://doi. org/10.11591/ijece.v6i4.10213
13. Darehshoorzadeh A, Boukerche A. Underwater Sensor Networks: A new challenge for opportunistic routing protocols. Communications Magazine, IEEE. 2015; 53(11):98-107. https://doi.org/10.1109/ MCOM.2015.7321977

14. Javaid N, Jafri M, Ahmed S, Jamil M, Khan Z, Qasim U. Delay-sensitive routing schemes for underwater acoustic sensor networks. International Journal of Distributed Sensor Networks. 2015; 11(3):1-13. https://doi. org/10.1155/2015/532676

15. Coutinho RW, Boukerche A, Vieira LF, Loureiro AA. GEDAR: Geographic and opportunistic routing protocol with depth adjustment for mobile underwater sensor networks. IEEE International Conference on Communications (ICC); 2014. p. 251-6. https://doi.org/10.1109/ICC.2014.6883327

16. Sun E, Shen X, Chen H, Wang C. Adaptive deployment scheme and multi-path routing protocol for WMSNs. Indonesian Journal of Electrical Engineering and Computer Science. 2014; 12(2):1454-61.

17. Ayaz M, Abdullah A, Faye I, Batira Y. An efficient dynamic addressing based routing protocol for Underwater Wireless Sensor Networks. Computer Communications. 2012; 35(4):475-86. https://doi.org/10.1016/j.comcom.2011.11.014

18. Wahid A, Lee S, Kim D, Lim KS. MRP: A localization-free multi-layered routing protocol for Underwater Wireless Sensor Networks. Wireless Personal Communications: An International Journal. 2014 Aug; 77(4):2997-3012. https:// doi.org/10.1007/s11277-014-1690-6

19. Al Salti F, Alzeidi N, Arafeh BR. EMGGR: An EnergyEfficient Multipath Grid-Based Geographic routing protocol for Underwater Wireless Sensor Networks. Wireless Networks. 2017; 23(4):1301-14. https://doi. org/10.1007/s11276-016-1224-0 